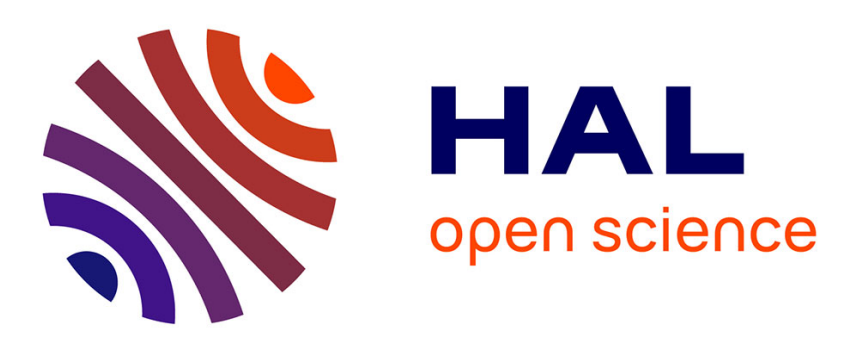

\title{
L'œuvre de Georges Hébert au Brésil et en France dans les écrits sur l'Education physique. Deux facettes de la nature (1909-1957)?
}

\author{
Jacques Gleyse, Carmen Lucia Soares, André Dalben
}

\section{To cite this version:}

Jacques Gleyse, Carmen Lucia Soares, André Dalben. L'œuvre de Georges Hébert au Brésil et en France dans les écrits sur l'Education physique. Deux facettes de la nature (1909-1957)?. Sport History Review, 2014, 45 (2), pp.171-199. 10.1123/shr.2013-0014 . hal-01334056

\author{
HAL Id: hal-01334056 \\ https://hal.science/hal-01334056
}

Submitted on 20 Jun 2016

HAL is a multi-disciplinary open access archive for the deposit and dissemination of scientific research documents, whether they are published or not. The documents may come from teaching and research institutions in France or abroad, or from public or private research centers.
L'archive ouverte pluridisciplinaire HAL, est destinée au dépôt et à la diffusion de documents scientifiques de niveau recherche, publiés ou non, émanant des établissements d'enseignement et de recherche français ou étrangers, des laboratoires publics ou privés. 
Jacques Gleyse is with the Faculté d'Education, Université de Montpellier 2 - EPS, Montpellier, Hérault, France. Carmen Lúcia Soares and André Dalben are each with the Department of Education, UNICAMP, Campinas, Sao Paolo, Brazil. Address author correspondence to Jacques Gleyse at jacques.gleyse@orange.fr.

\title{
L'œuvre de Georges Hébert au Brésil et en France dans les écrits sur l'Education physique. Deux facettes de la nature (1909-1957)?
}

\author{
Jacques Gleyse, ${ }^{1}$ Carmen Lúcia Soares, ${ }^{2}$ and André Dalben ${ }^{2}$ \\ ${ }^{1}$ Université de Montpellier 2 - EPS; ${ }^{2}$ UNICAMP
}

\section{Introduction}

La recherche qui suit, ayant la forme d'une épistémologie historique ou d'une archéologie au sens foucaldien du terme, met en relation la réception de l'œuvre de Georges Hébert au Brésil et en France dans les écrits concernant l'Education physique et sportive. Les textes étudiés, à cet effet, sont à la fois des textes officiels, des écrits parus dans des revues largement diffusées dans le champ, des journaux « grand public », des ouvrages portant sur les méthodes d'Education Physique et des ouvrages historiographiques concernant l'Education physique et sportive. De nombreuses archives, qui seront détaillées plus loin, ont servi de support à cette recherche bilatérale dont les bornes temporelles sont définies par la première importante publication de Georges Hébert (1909) et la date de sa mort ainsi que la parution du dernier tome de l'Education physique, virile et morale par la Méthode Naturelle (1957). L'idée centrale du texte est de permettre de percevoir au travers de ce travail d'archive, de cette « rétrodiction », comment la divergence possible entre deux visions de la «nature », joue un rôle important, au-delà, bien sûr, de la nationalité de l'auteur de la Méthode Naturelle.

Dans cette optique, il faut savoir qu'un important débat (fédéral) concernant l'institutionnalisation de l'Education Physique a lieu au sein de la société brésilienne (urbaine) dans les années 1920. Ce débat porte essentiellement sur l'intérêt des gymnastiques européennes. Cependant, celui-ci est aussi issu de la volonté de créer une méthode nationale d'Education physique, position soutenue, entre autres, par l'Association Brésilienne d'Éducation (ABE), société créée en 1924, dont la 
structure intègre une section Education Physique. Cette Association souhaite prendre ses distances avec l'influence militaire en Education physique, influence représentée, pour elle, par l'Education physique Méthode française (intégrant une part d'hébertisme).

Dans ce pays, les années 1920 sont, en ce sens, fondamentales pour comprendre l'institutionnalisation de l'Education physique, ainsi que la mise en place d'un ensemble de lois qui la rendent, à terme, obligatoire dans tout le pays. A titre d'exemple, on peut citer la loi promulguée par le Ministère de la Guerre, en 1929, sur les bases du travail d'une Commission composée, non seulement de militaires, mais, aussi de civils, issus de diverses institutions : médecins, avocats, ingénieurs, sportifs, députés. Cette Commission, cependant, reste présidée par le Ministre de la Guerre. Sa principale tâche est d'élaborer un avant-projet de loiprogramme règlementant l'enseignement de l'Education physique sur le territoire fédéral. Il est important de mentionner ce projet, car, son contenu est structuré sur la base d'une expérience réalisée au Centre Militaire d'Education physique créé en 1922 à Rio de Janeiro, sous l'influence de la Mission Militaire Française. Il y a une forte volonté du pouvoir de diffuser et, surtout, d'appliquer le "Règlement Général d'Education Physique", version brésilienne, en quelque sorte, de la "Méthode française".

A l'article 41 de ce Règlement: "Les Dispositions Transitoires", on peut lire l'affirmation suivante :

“ Tant que la Méthode Nationale d'Education physique brésilienne ne sera pas créée, la Méthode française sera adoptée dans tout le pays, sous le nom de 'Règlement Général d'Education physique'"'1.

Il est important de souligner, ici, l'influence de l'Armée française dans la modernisation de l'Armée brésilienne qui motive, sans aucun doute, l'adoption du Règlement $\mathrm{n}^{\circ}$ 7, Méthode française, destiné au corps d'Armée, modèle étendu également à la population civile ${ }^{2}$. Cependant, cette influence militaire est vivement discutée ainsi que l'atteste l'étude de Meily Assbú Linhales ${ }^{3}$, analysant de façon détaillée les positions des défenseurs d'une Education physique civile.

Deux études quasi identiques, sur ce sujet, ont été publiées par Thierry Terret et Leomar Tesche, l'une dans un ouvrage collectif dirigé par Gertrud Pfister, en 2010 (Gymnastics, a Transatlantic Movement - From Europe to America), sous le titre : French Gymnastics in Brazil : Dissemination, Diffusion and Relocalisation, l'autre dans International Journal of the History of Sport, en 2009, portant le même titre. Ces deux textes s'intéressent principalement à la diffusion des méthodes européennes, françaises et allemandes, dans l'extrême sud du Brésil, mais aussi à la diffusion de la « Méthode française » par les officiers de l'école de Joinville auprès de leurs homologues du sud Brésil. Ils montrent, à la fois, une volonté de reprise, mais également un refus des brésiliens d'appliquer telle quelle la Méthode française. Mais ces textes ne traitent qu'implicitement de la Méthode Naturelle de Georges Hébert, au travers du filtre de la «Méthode française». On voit, en tout état de cause, qu'il y a désir de transformation de la méthode proposée par les officiers français et de la Méthode française en particulier, pour en faire au plus vite une méthode plus spécifiquement adaptée au «tempérament» brésilien et au pays luimême.

On retrouve cette discussion au sein de l'Association Brésilienne d'Éducation $(\mathrm{ABE})$, mais aussi d'autres associations telle que la Société Brésilienne d'Hygiène 
(SBH), créée en 1919. Cette société, qui valorise les effets d'une pratique régulière d'exercices physiques, formule ses propositions en s'appuyant, au plan théorique, sur des traités de gymnastique française, suédoise ou allemande ${ }^{4}$.

Sur le thème des influences européennes, les études sont pléthore au Brésil. Les travaux de Carmen Lúcia Soares ${ }^{5}$; Victor Andrade de Melo ${ }^{6}$; Silvana Vilodre Goellner; ; Amarílio Ferreira Neto; Andrea Moreno; Ana Márcia Silva, Célia Guimarães Perini e Márcia de Souza Pedroso Agostini ${ }^{10}$; Meily Assbú Linhales ${ }^{11}$; Evelise Amgarten Quitzau ${ }^{12}$ ont étudié ce processus. Le dernier travail cité, se centre sur l'importance des méthodes de gymnastique européennes dans le processus d'institutionnalisation de l'Education physique brésilienne. Il démontre les influences médicales, militaires, pédagogiques reposant elles-mêmes, sur des fondements scientifiques.

Par ailleurs, même si l'étude proposée ici se centre plutôt sur cette zone, il faut préciser que le sud du Brésil, n'est pas le seul à être visé par ce processus. D'autres régions du pays, on le verra plus loin, touchées par une urbanisation rapide, sont également concernées. En effet, dès que se dessinent les caractéristiques d'une véritable ville, on trouve des tentatives d'institutionnalisation de l'Education physique sur la base d'influences européennes. C'est ainsi que différentes institutions, parmi lesquelles l'Ecole (les dirigeants qui en ont la charge), soulignent les bénéfices d'une Education Physique, établie sur ces fondements, surtout à partir de l'étude des méthodes de gymnastiques militaires ou pédagogiques.

En France, comme on le sait, le monde de l'Education physique, dans l'entredeux-guerres, se partage entre trois institutions puissantes :

— l'Instruction publique où a longtemps dominé Georges Demenij (1850-1917),

- la médecine où Fernand Lagrange puis Georges-Auguste Racine, Fernand Sandoz et Maurice Boigey apposeront leur marque,

—l'Armée et/où l'Ecole de Joinville avec Georges Hébert (même si le règlement d'EP, du 21 Janvier 1910, s'appuie plutôt sur les thèses de Philippe Tissié 1852-1935) qui développeront l'idée de Méthode Naturelle.

Cette dernière méthode, ensuite, de manière plus ou moins forte, envahira l'Instruction publique (Projet de règlement général, Méthode française, de 1919-23 et Règlement général. Méthode française, de 1925-35, publiés par l'éditeur militaire Charles Lavauzelles et compagnie. L'expression Méthode française est toutefois à attribuer à Georges Demenij) et même le domaine médical. La Méthode Naturelle de Georges Hébert dominera de manière écrasante les pratiques et les conceptions d'Education Physique à partir de son triomphe au Congrès International d'Education Physique de Paris, en 1913 et jusqu'aux instructions officielles du 20 juin 1959.

\section{La nature, mais laquelle?}

Lorsque l'on s'intéresse à l'idée de nature, en général, à l'extrême fin du XIXe et au début du XXe siècle au Brésil et en France, deux visions distinctes s'offrent au regard de l'historien. En France, la nature est désirée par les populations urbaines, qu'elles soient populaires ou bourgeoises, ayant récemment quitté leur campagne natale pour venir travailler dans les villes ${ }^{13}$. Le « sauvage » est considéré comme un modèle corporel positif perdu sous l'impact de l'industrialisation et de l'urbanisation 
mais aussi, à l'inverse, comme une race inférieure. C'est ainsi, par exemple, que le baron Pierre de Coubertin décrit les Noirs qui, selon lui, ne pourront jamais battre la race blanche aux Jeux Olympiques. Francis Galton, en Angleterre, ou Alexis Carrel et Charles Richet (Nobels de médecine en 1912 et 1913) en France, défendent des positions eugénistes assez similaires ${ }^{14}$. Il y a donc à la fois fascination et répulsion, désir et rejet ${ }^{15}$.

Au Brésil, les immigrés européens qui arrivent au début du XXe siècle dans la région sud et sud-est, région au climat tempéré, aux saisons bien marquées (bien loin du pays tropical, toujours ensoleillé et caricaturalement évoqué comme l'unique Brésil), se retrouvent dans des conditions de vie proches de l'Europe du sud. Ces immigrés sont le plus souvent des paysans pris, fréquemment, dans un rapide processus d'industrialisation induisant également un regard nostalgique sur la nature. Mais ils peuvent aussi se retrouver dans une campagne hostile produisant une vision opposée de la nature. Celle-ci est vécue comme dangereuse, peuplée de tribus indiennes hostiles (les Xavantes, par exemple) ou d'animaux sauvages agressifs (jacare, oncas pintadas, sucuris ${ }^{16}$ ). Une image stéréotypale prend place, également — dont nous reparlerons plus loin — celle de Jeca Tatu, paysan apathique, malade des fièvres, fainéant ${ }^{17}$.

Dans les villes brésiliennes, du début du XXe siècle (moment de l'institutionnalisation de l'Education Physique), tout comme en France, une nature urbanisée aseptisée, "hygiénisée » est fabriquée par les élites financières et intellectuelles, cultivées, sur un modèle pensé comme européen. Des idées et des propositions bien proches de celles existant en Europe sont formulées.

Pour ce qui concerne l'œuvre d'Hébert et surtout sa fécondité dans les écrits concernant l'Education Physique au Brésil, un paradoxe réside dans le fait que même si ses idées se basent sur la même nature éducatrice, bonne et généreuse, son nom et ses publications ne sont que très rarement mentionnés. D'ailleurs, y compris dans l'historiographie, l'étude: «Georges Hébert e o método natural: nova sensibilidade, nova educação do corpo ${ }^{18}{ }^{18}$ est encore aujourd'hui l'unique travail spécifique publié au Brésil.

Comme cela a été dit plus haut, le rapport fantasmé à deux natures bien différentes, l'une sauvage du Brésil rural, l'autre artificielle du Brésil urbain est peut-être l'un des éléments explicatifs (on ne peut pas nier que la nationalité du Capitaine de vaisseau joue évidemment, un rôle décisif) d'une fécondité différente de la Méthode Naturelle d'Education Physique dans ce pays et en France.

Dans le domaine de l'Education Physique, en France, le parangon de la dynamique «naturiste », est bien sûr: Georges Hébert (1875-1957), et surtout le contenu de son œuvre connu sous le terme de : "Méthode Naturelle " (le titre de plusieurs ouvrages est: L'Education physique virile et morale par la Méthode Naturelle). Mais, bien sûr, cette œuvre, dans un début de mondialisation (surtout au travers des processus de colonisation militaire et culturelle), ne reçoit pas la même audience et le même intérêt en Europe et ailleurs. Elle est assez populaire dans les Opera Balilla en Italie fasciste dans les années trente. On en trouvera des formes germaniques dans les films de Leni Riefenstahl par exemple : Olympia ou dans ses reportages photographiques sur l'Afrique et notamment les Noubas du Soudan mais, évidemment, sans aucune référence à un auteur français (l'ennemi de l'époque). L'Angleterre dit vouloir en faire la méthode officielle de la Marine après le congrès international d'Education Physique de Paris, en 1913. 
Elle devient la méthode officielle en France et en Belgique à partir de 1923 (Projet de règlement d'Education Physique Méthode française) et peut-être même un peu avant (dès 1919, tome 1 du projet). Elle triomphe au congrès International d'Education Physique de Paris en mars $1913^{19}$. Au Brésil, elle connaîtra une fécondité et un sort assez différents.

\section{France et Brésil urbains ou ruraux}

\section{Attraction et répulsion}

Le Brésil rural, au début du XXe siècle, est paradoxal. Il attire et repousse. Il est menaçant, en raison de pénuries, de préoccupations et de peurs justifiées. La nature qui éduque, régénère, fortifie le corps et l'esprit n'est pas celle de ce Brésil rural dont les références sont souvent associées aux images de vices, de maladies, de dégénérescence, de faiblesse de caractère, d'apathie et aux affres de la misère ${ }^{20}$ (il existe à partir de 1914 dans le journal O Estado de Sao Paulo : l'image négative de Jeca Tatu $)^{21}$.

A l'inverse, comme on vient de le voir, la vision positive de la nature est développée au sein d'un Brésil urbain à partir de la Proclamation de la République, en 1889, et est fortement influencée par les idées et les idéaux européens de l'époque. Le Brésil urbain du tournant du XXe siècle est différent de celui des champs, c'est le Brésil des populations laborieuses, prolétariennes mais aussi bourgeoises, de l'univers industriel, envahi par les fumés et les ombres tel que l'a décrit Maupassant, pour la France.

\section{$\backslash$ Insert Figure $1 \backslash$}

Le tout début du XXe siècle, dans ce pays, est fertile pour ce qui est de la référence à la nature et à ses constituants. La nature (fabriquée par l'homme) est perçue par les élites urbaines comme le lieu le plus adéquat d'éducation. C'est un moment d'ébullition culturelle où fleurissent différents projets favorisant le développement du pays. Une Education Physique dans la nature, des pratiques corporelles et sportives au grand air, font partie du projet des élites urbaines.

Dans la ville de São Paulo, une des expressions de ce mouvement vers la vie au grand air est pilotée par Mario de $A_{\text {ndrade }} 22$. Il a pour corollaire la création de parcs et jardins et la mise en œuvre d'une éducation des enfants proche d'une « nature » recréée et adaptée aux valeurs urbaines. Il est aussi important de souligner l'existence d'une école de plein air qui suit les modèles européens dessinés par l'Ecole nouvelle ${ }^{23}$. Dans le même temps, une culture sportive est née non seulement dans la ville de São Paulo, mais, aussi dans toutes les zones urbanisées du Brésil ou en cours d'urbanisation.

Dans la ville de São Paulo, au début du XXe siècle, les clubs et les associations sportives s'installent au bord des fleuves et des rivières qui baignent la ville, et mettent en place un ensemble d'activités sportives et récréatives aquatiques, championnats de natation, d'aviron, de voile, associés à des activités bucoliques, pique-niques et promenades se déroulant sur les rives des cours d'eau.

Dans la ville de Rio de Janeiro, alors capitale fédérale du Brésil, on peut voir de grandes transformations architecturales répliquant le modèle du Paris haussmannien avec de grandes avenues, des parcs, des jardins. Ces transformations sont contemporaines d'incitations aux pratiques corporelles et sportives au grand air, 
particulièrement en bord de mer : aviron, régates et natation ${ }^{24}$. Il est aussi important de signaler, bien plus tôt, la création du Jardin Botanique, en 1808, avant même de la proclamation de la République, en 1889.

Belo Horizonte, ville construite pour héberger la capitale de l'Etat de Minas Gerais, expression d'un projet architecturel influencé par la pensée hygiéniste, aura, elle aussi, de grandes avenues, des parcs et des jardins situés en bordure d'un lac artificiel où les sports et les pique-niques sont légion ${ }^{25}$.

Dans le sud du Brésil, la ville de Curitiba, capitale de l'Etat du Paraná témoigne aussi de ce moment d'engouement pour la nature fabriquée, artificielle. Il donne lieu à différentes initiatives telle que la création d'un jardin public dénommé : Passeio Público, conçu a partir des modèles de jardins européens et comportant, comme à Belo Horizonte un vaste plan d'eau artificiel. Dans cette ville, on assiste également à l'utilisation des fleuves et rivières pour des pratiques sportives et récréatives ${ }^{26}$.

La ville de Porto Alegre est touchée par des initiatives semblables sur les berges du grand fleuve Guaiba comme en atteste, entre autres, le travail, de Joana Carolina Schossler ${ }^{27}$.

Dans la région amazonienne, dans ce Brésil tropical - souvent imaginé, par les penseurs européens, comme le seul existant — la ville de Belém, est un exemple particulièrement frappant de ce rapport à une nature fabriquée de toute pièce. Lorsque cette ville vit l'apogée du caoutchouc, entre 1900 et 1920, elle se voit surnommée : «la petite Paris » et aujourd'hui encore, cette ville garde les traces d'une architecture «Art Nouveau ». Au cours de la période étudiée ici, la citée a vu le développement d'une véritable culture sportive sur les berges de ses fleuves et rivières (le Para entre autres). Les régates, les compétitions de natation et les clubs d'aviron, y fleurissent ${ }^{28}$.

Les exemples sont pléthore.

On peut donc affirmer que le Brésil urbain du début du XXe siècle voit l'essor d'une culture corporelle et sportive et que la nature y est constamment évoquée, par ses diffuseurs, comme lieu d'éducation et de régénération sanitaire et prophylactique. Pour autant l'œuvre de Georges Hébert ne semble à aucun moment servir de base à ces propositions.

Cependant, la nature proposée comme base d'une éducation du corps n'est pas une véritable «nature sauvage », en tout cas pas celle du Brésil de la selva (jungle) d'Amazon, du Mato-Grosso, de Goias. Cette nature des bois et des champs est considérée comme, à la fois «non-civique » et « non-civilisée ».

L'analyse détaillée de la réception de l'œuvre de Hébert au Brésil, au travers des archives et bibliothèques de plusieurs Facultés d'Education Physique tel que le Centre de Mémoire de l'Education Physique et Sport (CEME - Universidade Federal do Rio Grande do Sul); la bibliothèque de la Faculté de l'Education Physique (Universidade de São Paulo - USP) et de la Faculté d'Education Physique (Universidade Estadual de Campinas - Unicamp), permet de percevoir comment une partie de l'élite intellectuelle et gouvernementale brésilienne, citadine, s'est plus ou moins servie des idées hébertistes, d'une façon explicite ou implicite et le plus souvent sans référence à l'auteur lui-même. L'étude des archives montre, par ailleurs, le faible intérêt de l'historiographie brésilienne de l'Education Physique et du Sport pour l'œuvre de Georges Hébert.

On sait par ailleurs, que si Georges Hébert a visité, au cours de ses voyages transatlantiques, les Caraïbes (on connaît l'épisode de St Pierre au moment de 
l'explosion de la Montagne Pelée lorsque le croiseur Suchet sur lequel il est embarqué comme enseigne de vaisseau, mouille le 7 mai 1902, dans la baie), Cuba, la Colombie, le Venezuela ${ }^{29}$, il n'est jamais allé au Brésil et les archives brésiliennes ne contiennent aucune correspondance épistolaire avec ses homologues brésiliens dans le domaine de l'éducation et de l'Education Physique et a fortiori médical. C'est, entre autres, pour cette raison que l'étude proposée porte sur l'analyse des ouvrages, revues et de l'historiographie de l'Education physique et non sur d'autres éléments d'archives.

\section{La nature française moins ou plus "civilisée »}

Georges Vigarello et Michèle Métoudi dans un article intitulé « La nature et l'air du temps » (1980) tout comme André Rauch dans ses ouvrages : Le Souci du corps ou Vacances et pratiques corporelles (1983), permettent de comprendre le rapport relativement différent que la France populaire et bourgeoise entretient avec la notion de nature. La nature des Français est alors une nature totalement idéalisée liée à l'origine paysanne des classes ouvrières et des populations urbaines. En effet, la France est en train d'opérer alors sa véritable révolution industrielle et, surtout, urbaine. Il y a donc pour ces populations nouvellement intégrées dans les villes ou dans leurs banlieues, une profonde nostalgie de la nature. C'est cette nostalgie qui portera au pinacle la Méthode Naturelle de Georges Hébert. Celui-ci d'ailleurs s'appuie fortement sur ce fantasme nostalgique. Ce ne sont donc pas, contrairement au Brésil, les seules élites urbaines qui sont dans une position nostalgique au regard de la nature, ce sont aussi les classes populaires devenues récemment urbaines. De plus la nature qui va être utilisée n'est pas seulement une nature artificielle reconstituée dans les villes mais, quand cela est possible, une nature rurale « sauvage» : les bois, les forêts et les champs proches des villes (l'Ecole des Roches est à la campagne et non à Paris).

Mais il y a aussi une autre vision des choses. La France est alors un colonisateur forcené de l'Afrique Occidentale, Equatoriale et d'autres parties de la planète (Indochine, Afrique du Nord...). Les « sauvages » sont alors vus comme des modèles de corps à l'état natif, préservés de la civilisation, en quelque sorte non pollués par elle. A cette époque l'on parle cependant plus volontiers de dégénérescence de la race que de pollution. C'est pour cela que Hébert présente les photographies d'africaines ou d'africains dans de nombreux ouvrages (tout comme il présente par tradition gymnastique des images de la statuaire grecque).

\section{$\backslash$ Insert Figures 4 and $5 \backslash$}

Il s'inscrit par ailleurs, comme cela a été dit plus haut, dans la même logique eugéniste (bien que rien ne laisse penser qu'il fût eugéniste négatif comme eux) initiée par Francis Galton à la fin du XIXe siècle reprise par deux prix Nobel consécutifs, très cités. Le premier est proche du monde de l'Education Physique : Charles Richet (La Chaleur humaine), prix Nobel 1913. Le deuxième est davantage caractérisé par une proximité politique avec l'Action Française et le Parti Populaire Français de Doriot : Alexis Carel, prix Nobel 1912, qui publiera, plus tard L'Homme cet inconnu (1935). Tous ces auteurs croient que la civilisation industrielle conduit à la dégénérescence de la race blanche. Ils prônent, contrairement à Georges Hébert, un eugénisme négatif où il conviendrait de sélectionner les meilleurs types corporels. Galton, pour sa part, aurait limité cela au type corporel de la haute bourgeoisie 
anglaise voire londonienne. Georges Hébert, tout comme son prédécesseur dans le domaine: Georges Demenij, prône un eugénisme positif. Selon ce dernier, l'Education physique améliore la race française et par voie de conséquence la descendance des pratiquants assidus, d'où l'intérêt porté aux plus faibles et le modèle du « bon sauvage ».

On comprend bien que la situation ne saurait être la même dans un Brésil presque vide où la selva (la forêt primaire) est partout à portée de main où les animaux dangereux et les fleuves peu sûrs sont légions et où le paysan est considéré (Jeca Tatu) plutôt comme un dégénéré par les élites urbaines qui promeuvent la gymnastique, davantage d'ailleurs que l'Education physique, au sens hébertiste du terme. Le fantasme positif de la véritable nature y est forcément moins présent et on lui préfère, évidemment, une autre nature artificielle et urbaine.

\section{Un autre regard porté sur la nature}

Dès le début de son œuvre l'Etat et le monde journalistique s'intéressent aux propositions de Georges Hébert, en France. L'ouvrage L'Education Physique ou l'entraînement complet par la Méthode Naturelle, paru en 1912 mais réédité régulièrement, dans son édition de 1942 consacre 170 pages aux articles de journaux à grande diffusion, de l'Académie de médecine, de l'Académie des sciences, d'ouvrages, d'actes de colloques et de revues diverses consacrés à l'œuvre de Georges Hébert, articles parfois critiques mais le plus souvent panégyriques ${ }^{30}$.

Ainsi, le journal Le Figaro du 18 mars 1913 explique :

«Or ce sera l'honneur et la gloire du Lieutenant Hébert d'avoir emprunté aux sports ce qu'il fallait pour créer une méthode nouvelle dite naturelle, qui s'adapte d'une façon remarquable au tempérament national. [...] Les résultats obtenus par le Lieutenant de vaisseau Hébert sont prodigieux. [...] La méthode Hébert est d'une application facile ; on peut l'introduire, sans rien changer de ce qui est, dans l'éducation de toute la jeunesse française, à l'école au lycée. Qu'on le fasse ! Ce sera un bienfait national ».

Et effectivement cette proposition faite par ce journal généraliste, sera très vite mise en œuvre dans les règlements scolaires et dans les Instructions Officielles. On va y revenir plus loin.

Le journal L'Automobile, du même jour, est tout aussi dithyrambique lorsqu'il explique que : «Voici les pupilles de la Marine, buste, jambes, pieds nus. Sous la direction du Lieutenant de Vaisseau Hébert qui fut le véritable héros de cette belle fête du muscle, ils exécutèrent puis après eux les mousses et les fusiliers, les mouvements de la Méthode Naturelle. C'est eux qui nous donnèrent, avec leur peau bronzée par le grand air, leur allure souple et féline, leur musculature puissante et bien en relief, la plus forte impression d'art et de vigueur de cette journée renouvelée de l'Antique "».

Même le prestigieux journal Le Temps du 19 Mars 1913 écrit que : «Ce fut alors le clou de la journée, le triomphe d'un modeste et d'un acharné, le lieutenant Georges Hébert. Nous avons parlé mainte fois de la méthode rationnelle, de la Méthode Naturelle d'Hébert, nous avons dit les bienfaisants effets, la simplicité, l'emprunt qu'elle fait aux gestes instinctifs de l'enfant, et enfin les résultats obtenus par l'exercice pratiqué au grand air, jambes et torse nus. Mais il manquait au public d'avoir pu constater par lui-même ces réalités. Il est satisfait maintenant, car ce fut 
un enthousiasme unanime qui se manifesta après les démonstrations des pupilles, des mousses et des fusiliers marins ».

Mais ces journaux grand public ne sont pas les seuls à valoriser la Méthode Naturelle d'Education Physique puisque des revues très spécialisées ainsi que le monde médical en font de même à la même époque à quelques jours ou semaines près.

\section{L'œuvre de Georges Hébert au Brésil et en France dans l'historiographie}

L'analyse des principaux livres lusitanophones d'histoire de l'Education Physique et du sport au Brésil, concernant la période de l'étude, a été réalisée. Ce qui frappe, immédiatement, c'est la faible présence explicite de l'œuvre d'Hébert, ou, le faible nombre de lignes consacrées à son œuvre. En effet, dans le repérage de ces sources, aucun ouvrage écrit par des auteurs brésiliens portant en totalité sur la Méthode Naturelle d'Education Physique ou sur son créateur, Georges Hébert n'est présent. Les traductions intégrales en portugais des ouvrages n'existent pas.

Quelques très rares auteurs font références à son œuvre, mais ce sont le plus souvent des passages très bref. Le livre Histórico da Educação Física ${ }^{31}$, de Laurentino Lopes Bonorino et ses collaborateurs, rédigé en 1931, considéré comme la première publication spécifique de ce genre de recherche au Brésil, c'est-à-dire, comme un écrit sur l'histoire de l'Education physique y fait une très rapide allusion. La préoccupation centrale de ces auteurs est tournée vers les aspects historiques de la gymnastique en tant que forme de «l'éducation du physique », prenant sens dans un contexte mondial. C'est donc dans ces pages que l'on retrouve quelques indications descriptives de l'œuvre de Georges Hébert et de la Méthode naturelle, incluses dans le chapitre concernant à Gymnastique française. Dans le même temps où les auteurs consacrent une quantité considérable de pages (plusieurs dizaines) aux méthodes développées par Amoros et Demenÿ ${ }^{1}$, pour ce qui concerne Georges Hébert on ne peut trouver que dix pages de descriptions et de critiques.

Bonorino et ses collaborateurs utilisent pour leur ouvrage, les divisions classiques de périodes historiques et mentionnent la Méthode Naturelle de Georges Hébert au cours de la période contemporaine dans le cadre de leurs explications et analyses concernant la gymnastique française. Dans ces pages on trouve une très brève description de la méthode et des bases théoriques de la méthode Hébert mais toujours intégrées à une critique de ce qui est considéré comme les failles de la méthode, dès son origine, c'est-à-dire l'absence de bases physiologiques et scientifiques. De ce fait, en fonction de leurs propos, on peut affirmer que le lecteur est conduit, de lui-même, à douter de cette méthode et même à rejeter des propositions aussi éloignées des canons scientifiques. C'est d'ailleurs le premier paradoxe de l'importation de la méthode Hébert au Brésil. Dans, l'historiographie on lui fait place mais le plus souvent pour la critiquer, fréquemment de manière virulente.

${ }^{1}$ Demenÿ est dans le texte de cet auteur écrit ÿ mais on sait depuis l'analyse de Jacques Gleyse (1995) réalisée sur la signature de Georges Demenij ainsi que grâce à son écriture en romain minuscule dans la préface de l'ouvrage du colonnel Lefébure que le nom, d'origine flamande s'écrit bien Demenij. 
D'autres aspects que les auteurs tiennent à signaler et à souligner concernent le fait qu'Hébert considère à égalité les sexes et que pour lui la supposée infériorité physique des femmes n'existe que parce qu'elles ne reçoivent pas la même Education Physique dès l'enfance et parce qu'elles ne sont pas confrontées aux mêmes défis physiques que les garçons et les hommes. Ils expliquent que Hébert pense que l'égalité des sexes deviendrait donc, possible à l'âge adulte, dans les mêmes conditions éducatives. Bonorino et ses collaborateurs disqualifient la position d'Hébert considérant que celle-ci ne s'appuie pas sur la physiologie de la femme et sur des bases scientifiques sérieuses, mais, simplement sur l'idéologie ${ }^{32}$.

Au contraire, l'ouvrage de Fernando de Azevedo, Da educação física: o que ela é, o que tem sido e o que deveria ser (1th. ed. 1916) ${ }^{33}$, dont un passage est consacré aux différences entre la gymnastique allemande et la gymnastique suédoise (chapitre 7), contient, dans ses derniers paragraphes, quelques brèves considérations sur la Méthode Naturelle de gymnastique. Dans ce livre, Fernando de Azevedo qualifie la gymnastique créée par Friedrich Ludwig Jahn d'anti-fonctionnelle et même de déformatrice et, cet intellectuel brésilien, en s'appuyant sur les études de Philippe Tissié, considère cette méthode comme antihygiénique, au regard de la gymnastique de Pier Henrich Ling. Ensuite, il affirme que la gymnastique aux appareils de Jahn est incorporée dans la gymnastique éclectique française (Méthode française) et que la Méthode Naturelle de Georges Hébert est très bien reçue en France en raison de ses exercices considérés comme moins analytiques et plus complets, synthétiques et utilitaires. Finalement, Azevedo met en relation la Méthode Naturelle avec l'éducation hellénique et recommande sa pratique comme un complément de la gymnastique suédoise, après la période de l'adolescence. Cet auteur construit ainsi des binômes intégrant la gymnastique suédoise et allemande, la Méthode Naturelle et la gymnastique française et se positionne en faveur de la gymnastique suédoise et de la Méthode Naturelle. Ses analyses prennent comme base, principalement ses études concernant à l'éducation grecque. Il est un des très rares auteurs, au cours de cette période, à valoriser partiellement la méthode Hébert au Brésil.

L'œuvre d'Inezil Penna Marinho, intitulée Sistemas e Métodos de Educação Física $^{34}$, étudiant les principales méthodes de gymnastique élaborées en Occident, consacre un chapitre de 60 pages à l'œuvre de Georges Hébert et à la Méthode Naturelle. Dans l'inventaire des sources cet auteur est celui qui, dans l'historiographie de l'Education physique au Brésil, consacre le plus grand nombre de pages à ce thème. Son contenu, essentiellement descriptif, porte sur les 4 tomes du livre L'Education Physique virile et morale par la Méthode Naturelle. Les relations très étroites d'Inezil Pena Marinho avec le gouvernement brésilien de la période : la dictature militaire de l'Etat nouveau ${ }^{35}$, peuvent expliquer la proximité avec la méthode officielle du régime de Vichy, sous Pétain (en France occupée), même si l'Estado Novo finira par s'engager avec les alliés dans la bataille contre le IIIe Reich.

Un autre livre ${ }^{36}$ fait référence directement à Georges Hébert, mais encore d'une façon critique : A harmonia entre o corpo e o espírito, de Nicanor Miranda, chef de la Division de Récréation du Département de Culture de Sao Paulo. Au contraire de Fernando de Azevedo, Nicanor Miranda disqualifie la Méthode Naturelle de gymnastique en prenant pour argument central le fait que l'enfant joue pour satisfaire une pulsion, un instinct primaire : jouer. Cet « instinct ludique », pourrait-on dire, ne peut pas le conduire à la pratique de la gymnastique. Ainsi le jeu 
a pour cet auteur une suprématie sur toute méthode de gymnastique construite ou fonctionnelle (comme la méthode Hébert). De plus, Miranda affirme que la gymnastique de Georges Hébert se base sur des «principes archaïques, anachroniques et sans aucun fondement scientifique $»^{37}$. Pour fonder son argumentation, à propos du jeu récréatif, Miranda s'appuie sur l'œuvre du pédagogue nord-américain John Dewey.

Cet auteur affirme, aussi, que la méthode Hébert : « valorise excessivement les exercices ou les mouvement dénommés : naturels $»^{38}$ et que la civilisation actuelle (1945), sans rapport avec l'homme primitif, doit être dans son temps : " penser une possible échappatoire au travers d'un retour au primitivisme est une illusion ${ }^{39}$. Et pour bien montrer son désaccord avec Hébert, à ce sujet, Miranda s'appuie sur l'ouvrage du physiologiste Français Maurice Boigey.

Cet inventaire des ouvrages évoquant l'œuvre d'Hébert, dans l'historiographie brésilienne de l'Education Physique, s'achève avec un texte orignal. Il s'agit de : Cultura Physica Feminina ${ }^{40}$, de Lotte Kretzchmar, directrice de 1'Institut Féminin d'Education Physique, ancien Institut Allemand, situé dans le centre de Rio de Janeiro. Ce livre attire l'attention par le fait qu'il est illustré par quelques photos extraites du livre: Muscle et Beauté plastique $e^{41}$. La première partie du livre, dans laquelle sont intégrées les photos, est avant tout une étude sur la beauté féminine classique. Les photos, qui apparaissent avec pour sous-titre le nom d'Hébert, ont pour but d'illustrer les "déformations » et « anormalités » du corps féminin par rapport à la statuaire grecque. Dans la deuxième partie de ce livre, l'auteur fait un récit des cours de «culture physique » dans cet Institut. Il n'y a aucune citation ou référence directe à l'œuvre de Georges Hébert, sauf les sous-titres des photos. L'auteur cite comme base pour ses études Platon, Socrate, Rousseau, Pestalozzi, Dalcroze et Demenij, jamais George Hébert.

Pour la partie française, le travail d'historiographie, beaucoup plus aisé, a été réalisé dans différents ouvrages et articles, de l'auteur même, et sur l'auteur ou la méthode. Il n'était donc pas absolument indispensable de retourner à des fonds d'archives spécifiques, si ce n'est les ouvrages de Georges Hébert lui-même ou des articles peu ou pas diffusés, dans la mesure où les travaux de Jean-Michel Delaplace $^{42}$, de Gilles Bui-Xuân \& Jacques Gleyse ${ }^{43}$, de Sylvain Villaret \& JeanMichel Delaplace ${ }^{44}$, de Pierre Arnaud ${ }^{45}$ ou de Gilbert Andrieu ${ }^{46}$ sur cet auteur sans compter les dizaines d'articles, parus ici ou là, et notamment dans les actes du congrès de l'I.S.H.P.E.S. de Montpellier (2001), consacré à la Nature, à l'Education Physique et au sport - sont des sources très fiables.

Le travail d'archive se limite donc à quelques articles de la revue l'Education Physique, de la revue Culture Physique, de la revue E.P.S. ainsi qu'aux ouvrages de Georges Hébert lui-même.

On sait par ailleurs que le fils de Georges Hébert, Régis Hébert, contacté par les auteurs de l'article, a toujours refusé l'accès direct de ses archives privées aux historiens de l'Education Physique et du sport. Il n'a pas été possible de les consulter et elles ne sont toujours pas déposées à la Bibliothèque Nationale de France. Quelques nouvelles informations ont cependant pu être recueillies lors du colloque de Reims concernant le $100^{\text {ème }}$ anniversaire de la création du Collège d'Athlètes les 3 et 4 octobre 2013 notamment l'absence de correspondance épistolaire avec les autorités brésiliennes.

A l'inverse des fonds brésiliens, ce qui est très compréhensible du fait que l'auteur est Français, on peut retrouver les ouvrages d'Hébert dans toutes les 
bibliothèques des Unités de Formation et de Recherche en Sciences et Techniques des Activités Physiques et Sportives (UFRSTAPS, anciennement Instituts Régionaux d'Education Physique puis Unités de Formation et de Recherche en Education physique et sportive à partir de 1969), dans de nombreuses bibliothèques universitaires (de médecine ou de sciences humaines), bien sûr, à la Bibliothèque Nationale de France et à la bibliothèque de l'Institut National des Sports et de l'Education Physique, encore aujourd'hui. On peut également trouver ces ouvrages dans les bibliothèques de l'Armée et à la vente, assez fréquemment, chez des libraires spécialisés dans les livres anciens. Cela témoigne de leur diffusion dans l'espace géographique français.

\section{Archives et bibliothèques consultées}

Pour la partie brésilienne de cet article, le travail d'archive a eu lieu à la Biblioteca da Faculdade de Educação Física da Unicamp et la Biblioteca da Faculdade da Escola de Educação Física e Esporte da USP (Sao Paulo), où il est possible de trouver quelques livres de Georges Hébert non traduits. Il est également important de dire que pratiquement l'ensemble des ouvrages de cet auteur est conservé dans le fonds dénommé : Centro de Memória do Esporte (CEME), hébergé par l'Université Fédérale du Rio Grande du Sud, dans la ville de Porto Alegre. La présence de ces ouvrages dans ces fonds est un indice important pour dire que les œuvres de Georges Hébert ont été connues au Brésil, surtout par les enseignants et les personnes intéressées par l'Education physique et le sport. Dans le même temps, ce qui semble très significatif et étonnant réside dans le fait de n'avoir aucune traduction complète de ces ouvrages en portugais, seulement de quelques articles (souvent des chapitres et parties d'ouvrages). Cela tend à prouver, à l'inverse, le faible intérêt que le champ de l'Education physique a eu pour la diffusion cette méthode au Brésil.

Le Ministre brésilien Monsieur de Magalhães, comme en témoigne le document du Congrès International d'Education Physique de Paris ${ }^{47}$, en 1913, est présent lors des démonstrations hébertistes. La commission brésilienne qui a également participé à ce Congrès est composée, majoritairement, d'acteurs du monde sportif tels que le Président de la Fédération Brésilienne de Sports Nautiques, les Directeurs du Club de Régates de Flamengo et le Président de ce même club de Football, d'un journaliste et de deux médecins. Aucun concepteur et acteur de l'Education Physique brésilienne n'est présent, au sein de cette commission.

En France, comme cela a été précisé plus haut, la plupart des archives sont très facilement accessibles et ont été étudiées dans diverses bibliothèques d'UFRSTAPS, à l'Institut National des Sports et de l'Education Physique à Paris ou encore dans des fonds spécialisés comme le Centre d'Etude, de Documentation et de Recherche en Histoire de l'Education de Montpellier. On peut y trouver la plupart des revues, par exemple l'Education physique, mais aussi tous les ouvrages de Georges Hébert ou encore des ouvrages portant sur l'œuvre d'Hébert.

On retrouve, notamment, de longs chapitres sur l'œuvre d'Hébert en particulier dans l'Encyclopédie des Sports, de 1924 ou dans le volumineux Traité d'Education Physique dirigé par Marcel Labbé, en 1930. De nombreux documents officiels font également référence à la Méthode Hébert : Le Projet de Règlement Général Méthode française de 1919-1923, le Règlement Général Méthode française de 1925-1934 et même les instructions officielles ministérielles du $1^{\text {er }}$ Juin 1941 ou encore celles de 1945 et 1959. 


\section{La réception de l'œuvre de Georges Hébert au Brésil et en France dans les journaux généralistes et les revues spécialisées}

$\mathrm{Au}$ Brésil, un important débat sur la nécessité de l'institutionnalisation de l'Education Physique en tant que discipline scolaire débute entre 1920 et 1940. Les traces de l'œuvre de Georges Hébert, dans les archives et la littérature, au Brésil ont été recherchées essentiellement au cours de cette période et au travers de ce débat. Il est possible de justifier également ce découpage temporel par la création, au Brésil, des premiers cours supérieurs (universitaires) d'Education Physique aussi bien que des premières structures officielles destinées à sa gestion publique. La période choisie se superpose, ainsi approximativement avec le moment où la méthode créée par Georges Hebert obtient une très importante notoriété en France. C'est à partir de ce découpage temporel qu'ont été recherchées les différentes sources concernant l'Education Physique et le sport telles que :

(a) des revues scientifiques,

(b) des revues de vulgarisation scientifique,

(c) des publications officielles,

(d) des annales de congrès,

(e) des livres et chapitres de livres publiés au Brésil et en France sur le sujet de l'Education Physique brésilienne.

Le repérage des sources a débuté par la revue Educação Physica dont 88 numéros publiés entre 1932 et 1945 ont été consultés. Selon Omar Schneider and Amarílio Ferreira Neto ${ }^{48}$, cette revue permet d'ébaucher un panorama acceptable des thèmes qui animent les discussions sur les contenus de l'Education Physique et sa place dans l'éducation brésilienne.

Il faut signaler, encore, comme cela a rapidement été évoqué plus haut, qu'une période de la publication coïncide avec le manque de liberté d'expression lié à la première dictature brésilienne du XXe siècle, nommée: "l'Etat Nouveau"49. C'est durant cette période que la revue acquiert réellement son statut de périodique scientifique. Toutefois il ne serait pas correct de la considérer comme un simple moyen de propagande idéologique ${ }^{50}$ car bien que cet aspect soit présent, la Revista véhicule aussi d'autres visions plus " pédagogiques » ou hygiéniques ${ }^{51}$.

Publiée par la Companhia Editora S.A do Rio de Janeiro, cette revue Educação Physica, a eu une importante diffusion territoriale, dans les différentes régions (estados) brésiliennes aussi bien que dans d'autres pays d'Amérique du Sud, d'Afrique Orientale et au Portugal. Dans l'inventaire produit par Omar Schneider and Amarílio Ferreira Neto, 3.768 articles dont 1.566 signés par 805 auteurs différents, ont été catalogués. A l'intérieur de ces pages sont présents, parmi d'autres nationalités, des auteurs Brésiliens, Nord-Américains, Français. A part les auteurs brésiliens (172 occurrences) ce sont les auteurs nord-américains qui sont les plus nombreux : 155. Les Français signent seulement 22 articles au cours de la période étudiée ${ }^{52}$.

Généralement, les articles publiés par des auteurs étrangers ne sont pas écrits spécialement pour la revue, mais, sont principalement constitués de traductions de parties de livres, de chapitres de livres, aussi bien que par des articles publiés dans des revues internationales. Il ne s'agit donc pas d'articles originaux. La réception de 
ce matériel et sa traduction en portugais est réalisée par des collaborateurs de la revue, qui, majoritairement, sont en lien avec l'Association de la Jeunesse Chrétienne (Associação Cristão de Moços-ACM), c'est la raison pour laquelle une grande partie des articles étrangers, traduits, proviennent des Etats-Unis d'Amérique du Nord (en contact institutionnel avec les Young Men's Christian Association (YMCA), les mêmes relations ont été établies en France concernant les Jeux Interalliés ${ }^{53}$ ).

Parmi les auteurs français traduits pour cette revue, on trouve le nom de Georges Hébert. Mais six articles, seulement ont été recensés ${ }^{54}$. Les cinq premiers, sont, en réalité, des chapitres du livre : Le Sport contre l'Education physique ${ }^{55}$, et le dernier semble être la traduction de parties de son ouvrage : La Culture virile et les devoirs physiques de l'officier combattant ${ }^{56}$. Dans tous les cas, la revue ne fait aucune référence aux ouvrages et ne dit pas d'où ces textes sont extraits. Une communication présentée en France en $2001^{57}$, lors du Colloque ISHPES de Montpellier, et développée dans une publication au Brésil en $2003^{58}$, faisait référence à ces sources. Cette communication orale a été publiée en $2004^{59}$, dans les actes.

Il est important de dire tout de suite que les textes sélectionnés par la revue Educação Physica ne prennent pas comme sujet principal les contenus de la Méthode Naturelle, mais, surtout les critiques formulées par Hébert concernant les excès, les dépassements des athlètes et spécialement de ceux ou celles qui gagnent leur vie avec les exhibitions corporelles de force et d'adresse.

La revue Educação Physica a publié, par ailleurs, la traduction d'un article de Edmond Desbonnet, écrit originalement dans la revue française La Culture Physique : «Crítica ao método natural de Georges Hébert 》 (Educação Physica 38, no.1 (1940): 33-36). Cet article apporte des arguments très critiques et même méprisants à l'encontre de Georges Hébert et de la Méthode Naturelle d'Education Physique. Il affirme que Georges Hébert n'a rien inventé, que ses propositions d'exercices sont des caricatures, qu'elles utilisent des processus barbares d'Education Physique. Cet article l'accuse, également, ad hominem, de ne pas pratiquer ce qu'il propose puisqu'il habite Paris et non la campagne et donc, ne peut certainement jamais, lui-même, se mettre nu pour réaliser les exercices physiques proposés. Le texte de Desbonnet, ne valorise donc pas du tout la Méthode Naturelle mais, au contraire, la disqualifie. La publication de cet article critique témoigne d'une forme de rejet de cette méthode par les éditeurs Brésiliens.

L'étude d'une autre revue: la Revista de Educação Física (1932-2010), publiée par l'Armée brésilienne, ne fait apparaitre aucune référence ni même une seule allusion à Georges Hébert. Au cours de la période, la majorité des articles sont écrits par des officiers de l'Armée brésilienne et, plus spécifiquement, par ceux qui sont alors professeurs à l'Ecole d'Education Physique de l'Armée. La diffusion nationale de la revue, au cours de la période, n'est pas circonscrite au milieu militaire, mais s'étend aussi aux civils. D’une façon plus générale, la revue s'est constituée comme un moyen de diffusion des doctrines militaires de l'Education Physique. Elle est destinée à la société brésilienne. Selon Berto e Schneider ${ }^{60}$ : « ce périodique tend, par son contenu, à devenir l'organe officiel et légitime de l'Education Physique scolaire ». En ce sens, l'absence de textes traitant des ouvrages et de la Méthode Naturelle de Georges Hébert, dans cette revue, semble être un très bon indicateur de la place réservée à la Méthode Naturelle, dans le champ de l'Education physique brésilienne, même si des bribes des techniques Hébert sont présentes dans la «Méthode française », dont il est très rarement 
question. Il est également intéressant de constater que dans le projet officiel destiné à l'Education physique brésilienne, forgé par l'Ecole d'Education Physique de l'Armée, ne sont pas incluses les théories d'Hébert, ou, du moins, qu'elles ne sont pas explicitement présentes.

L'Ecole d'Education Physique de l'Armée brésilienne est représentée, par ailleurs, aux trois principaux congrès d'Education Physique et de sport réalisés au cours des décennies 1930 et 1940 au Brésil :

(1) VII Congresso Nacional de Educação (RJ/1935);

(2) I Congresso Paulista de Educação Física (SP/1940);

(3) I Congresso Pan-Americano de Educação Física (RJ/1943).

Ces congrès ont donné lieu à des actes. L'analyse de cet ensemble de documents permet de constater l'absence de références à Georges Hébert. Il y a seulement des indications sur l'intérêt de la Méthode française (qui peut contenir des techniques de Méthode Naturelle) pour le développement de l'Education Physique scolaire au Brésil. Ces indications font référence au Règlement $n^{\circ} 07$, publié en 1931 (bien différent du Règlement général. Méthode française, de 1925, en France, fondé essentiellement sur la méthode Hébert), sur lequel repose la création officielle de l'Ecole de l'Education Physique de l'Armée. On affirme toutefois que la Méthode française ne saurait être appliquée telle quelle au Brésil. Elle doit impérativement être adaptée aux conditions structurelles, institutionnelle, climatiques locales et au tempérament national brésilien.

Dans la Revista de Educação, support de diffusion des actions du Secrétariat à l'Education et la Santé Publique, de Sao Paulo (Estado), nous avons trouvé très peu d'articles qui font référence spécifiquement à l'Education physique et au sport. Cependant il y a de nombreux articles concernant l'éducation hygiénique qui évoquent, même si c'est seulement de façon synthétique, l'importance de la nature dans l'éducation des enfants. Ces références, en général, soulignent la valeur de l'ambiance naturelle pour l'Education en s'appuyant sur les idées de Jean-Jacques Rousseau, idées centrales pour le mouvement de l'Ecole Nouvelle. Il est important de mettre en évidence que l'imaginaire de la nature, mis en scène dans ces textes officiels, n'évoque pas la spécificité de la nature brésilienne et les conditions de vie des habitants du monde rural, beaucoup décrits, pourtant, dans les rapports de voyages de médecins hygiénistes, comme, par exemple, Belisário Penna ${ }^{61}$.

Plus généralement, il est possible de constater que dans les sources concernant le champ de l'Education, à l'instar de revues scientifiques, de textes officiels et d'annales de congrès, la nature est décrite, en quelque sorte, de manière idéalisée et romantique. Le fait de souligner les vertus de la nature dans ces textes montre, nettement, qu'il s'agit d'une "nature urbaine », c'est-à-dire, une nature spécifique, créée de la main de l'Homme $^{62}$, bref, une reconstitution urbaine de la nature, une sorte d'invention de la nature.

Certaines études prennent pour base des rapports médicaux et les études scientifiques de santé publique et non les idées de Rousseau, mais, dans les deux cas, il n'y a aucune référence à Georges Hébert et à la Méthode Naturelle d'Education Physique.

Deux revues de vulgarisation scientifique brésiliennes, dans lesquelles des thèmes comme les exercices physiques, la gymnastique, le sport et la beauté 
féminine sont évoqués, ont été également étudiées. Il s'agit de la revue : Vida de Saúde (1938-2010), et de la revue : Viver! (1938-1942). Dans les éditions consultées sur la période étudiée, aucune référence à Georges Hébert et à la Méthode Naturelle n'est présente. On voit donc que la fécondité de la Méthode Naturelle de Georges Hébert au Brésil est plus que mitigée pour ne pas dire très faible.

Tout au contraire, l'évaluation de la méthode Hébert par les médias et les institutions françaises est dithyrambique. Même deux semaines après le congrès International d'Education Physique de Paris, et la prestation proposée, par le Lieutenant de vaisseaux, aux spectateurs, le 18 mars 1913, Le Figaro, Football, Le Fait de la semaine, L'Opinion, Le Cri de Paris, L'Intransigeant, Le Soleil, L'Echo des sports, l'Eclair, L'Univers, l'Auto, La Presse, Le Journal, Le Gymnaste, La Culture Physique, L'Action Française, bien sûr L'Education Physique et même L'Humanité (à l'autre bout du spectre politique comparé à l'Action Française), de Jaurès, réalisent un véritable panégyrique de la Méthode Naturelle.

Il faut insister sur l'article paru dans le dernier journal cité :

« J'ai vu un jour un admirable spectacle. J'ai vu une troupe d'enfants, qu'on m'avait dit être les fils de pauvres gens, courir, chanter, rire, jouer, en montrant librement à l'air leur petite poitrine dure et bronzée ; puis j'en vis d'autres, plus âgés au regard clair, au thorax puissant aux jambes vives, d'autres encore vinrent qui, ceux-là étaient presque des hommes et couraient, riaient, chantaient, jouaient, comme les plus jeunes. [...] Grand mince, serré dans sa tunique sombre aux épaulettes d'or, talonnant le plancher d'un pas souple et lent, modeste, sans geste inutile, la tête un peu inclinée, il regardait manœuvrer et vivre ses élèves. Et du vitrail la lumière pâle tombait sur les yeux d'apôtre, énergiques et rêveurs, du lieutenant Hébert. [...] Hébert a l'honneur d'apparaître comme le premier de ces éducateurs physiques dont parle Jaurès dans l'Armée nouvelle, qui élèveront, dans l'avenir tous les enfants fraternellement réunis, pour la seule beauté et la seule force de la nation. [...] saluons-le, socialistes; cet homme-là est digne d'être des nôtres et digne de notre idéal ${ }^{63}$

Même l'Académie Française sous la plume de Jean Richepin (qui avait participé à la création de la Ligue Française d'Education Physique en 1888) encensera la Méthode Naturelle le 5 mai 1913 dans le Journal de l'Université des Annales.

Des articles paraîtront également à l'étranger dans le journal The Times et quelques autres encore (surtout en Europe), aussi prestigieux. Autrement dit, d'autres pays, reçoivent positivement la Méthode Naturelle, mais il s'agit de vieux pays européens, industriels, en général.

De nombreux médecins dans Le Bulletin médical tels A. Thooris, Lanel, F Armand-Delille, font l'éloge scientifique et hygiénique de la Méthode Naturelle de Georges Hébert. Jusqu'au Professeur William R. Smith, qui lors du discours d'inauguration du Congrès du Royal Institute of Public Health, en mai 1913, regrette que cette méthode ne soit pas encore mise en œuvre au Royaume-Uni, même si, selon lui, l'amirauté songe à l'adopter pour la marine. C'est dire, vu l'importance de la marine et de l'Institut Royal d'hygiène, la puissance symbolique que représente alors la méthode présentée par Georges Hébert, sous le nom de Méthode naturelle d'Education Physique et sa fécondité, dans ce pays pourtant "ennemi héréditaire » de la France et peu francophone, contrairement au Brésil des élites. 
Bref, même si quelques critiques se font jour (on a vu précédemment celle de Desbonnet dans La Culture Physique, traduite en portugais), le consensus est quasi total. L'argumentaire est presque toujours le même pour valoriser cette méthode. Tout d'abord elle est joyeuse et agréable. Ensuite elle s'exécute largement dénudé. Les corps sont bronzés (il faut rappeler que le bronzage au XIXe et début du XXe siècle en France était suspect parce que paysan ${ }^{64}$ ). Les muscles sont fins et les pratiquants sont vifs. L'idée qu'elle se pratique également selon des normes naturelles et selon les pré-requis de la nature est presque toujours soulignée. Le fantasme de nature, associé à celle-ci, est donc largement positif, comme on l'a déjà vu plus haut.

\section{Les retombées institutionnelles}

En France, l'Armée s'approprie immédiatement la Méthode Naturelle (ce qui est logique puisque Hébert est un militaire). Plusieurs bataillons sont entraînés par Georges Hébert (qui sera grièvement blessé à Dixmude avec ses troupes) au cours de la première guerre mondiale, après la création, puis la destruction du Collège d'Athlètes de Reims. Ces bataillons, systématiquement entraînés par Hébert, à partir de 1916, subissent des pertes bien moindres que tous les autres bataillons jetés dans le conflit. A tel point qu'à l'issue du conflit, l'Armée adopte massivement la Méthode Naturelle, en premier lieu à l'Ecole de Joinville. Le général Mac-Mahon lui-même recommande la méthode. Le Grand Quartier Général fait savoir le 17 juin 1918, qu'il recommande l'entraînement par la Méthode Naturelle.

Même des entreprises privées paternalistes, telle Michelin à Clermont-Ferrand, recommandent la Méthode Naturelle dans ses écoles industrielles ${ }^{65}$.

C'est enfin l'école qui dès le $1^{\text {er }}$ Juillet 1919 s'empare de la Méthode Naturelle. En effet, le Projet de règlement général d'Education Physique qui est publié de 1919 à 1923 (selon les catégories d'âge), approuvé par le Ministre de l'Instruction Publique et des Beaux-Arts, comme complément du Manuel d'exercices physiques et de jeux scolaires organise tous ses contenus à partir des conceptions de la Méthode Naturelle. Les Instructions officielles du 20 juin 1923, destinées à l'enseignement primaire élémentaire, conseillent de s'appuyer sur le projet de règlement de 1919 pour structurer les contenus scolaires.

On retrouvera cette domination écrasante de la Méthode Naturelle aussi bien dans le tome consacré à l'enfance (1919), que dans celui consacré à l'Education Physique secondaire (pubertaire et post pubertaire), en 1922, ou dans le troisième tome traitant de l'Education Physique supérieure (sportive et athlétique).

Un peu plus tard, le Règlement Général d'Education Physique. Méthode française, publié en plusieurs tomes à partir de 1925 et jusqu'à 1935, va également promouvoir la Méthode Naturelle de Georges Hébert, y compris en structurant des contenus plus ou moins sportifs (Tome 2), selon les catégories de la Méthode Naturelle : «marcher, courir, sauter, grimper, lever, lancer, porter, quadrupédie, attaque-défense et nager $»$. Et bien que l'on puisse constater, sur ce dernier, point que l'apprentissage de la natation se fait encore à sec, contrairement aux préceptes de Georges Hébert, on peut dire que la Méthode Naturelle domine tous les tomes de ce monument Français de l'Education Physique de l'entre-deux-guerres.

D'autres ouvrages, comme cela a été rapidement évoqué plus haut (Encyclopédie des sports, Traité d'E.P.), qui se veulent être des sommes au cours de 
la période, font une place importante à la Méthode Naturelle, bien que les autres méthodes soient présentées.

On pourrait multiplier les exemples à l'infini. On doit par exemple souligner la proximité de la Méthode Naturelle avec celle prodiguée aux Hitler Jugends ou Jugends S.S. Bien entendu, ni d'un côté du Rhin ni de l'autre cette proximité ne sera revendiquée. Cependant, dès 1942, après l'occupation de la totalité de la France par le IIIe Reich, au sein des Chantiers de Jeunesse, le Guide d'entraînement physique, de 1942, prône la Méthode Naturelle comme contenu central de l'Education Physique. Certains auteurs affirmeront même que la Méthode Naturelle est le «massif central » ${ }^{66}$ de l'Education Physique Méthode française, sous le régime de Vichy.

Cependant Georges Hébert, lui-même, se désolidarisera de la rédaction des Instructions officielles du $1^{\text {er }}$ Juin 1941. Pourtant des passages entiers, consacrés à la Méthode Naturelle semblent être copiés de sa propre main. On y retrouve aussi des éléments qui ont dû être issus des conceptions très fortement naturalistes d'Ernest Loise $^{67}$ (Gleyse, 2012). Mais bien après cette période, encore, jusqu'aux instructions officielles du 20 Juin 1959, la Méthode Naturelle gardera une place de choix dans «L'Education physique méthodique», même si un certain recentrement sur la méthode néo-suédoise a pu avoir lieu à partir de 1959. Des articles, nombreux encore valoriseront la Méthode Naturelle d'Education Physique dans le tout début des années soixante, sous la plume de David, dans la revue $E P S$, trois ou quatre ans après la mort de Georges Hébert. C'est dire la puissance symbolique qu'eut cette vision de l'Education physique dans l'école et plus généralement dans la société française puisque c'est pratiquement un demi-siècle qui sera dominé par cette pratique. Progressivement, à partir des années soixante, les pratiques sportives stricto sensu se substitueront à la Méthode Naturelle, du moins pour les garçons.

Mais si nous revenons un peu sur ce qui a été décrit pour le Brésil, au cours de la période de la deuxième guerre mondiale, l'idéologie de L'Estado novo de Getulio Vargas, sa proximité avec le régime de Vichy, est susceptible d'expliquer la publication de nombres d'articles sur la Méthode Naturelle autour de la période 1941-1943, ainsi que le chapitre écrit par Inezil Penna Marinho sur la Méthode Naturelle d'Hébert, chapitre le plus long sur ce thème et cet auteur comme nous l'avons déjà vu. On peut en effet, supposer qu'un tel type de gouvernement s'inspirait idéologiquement des positions mussoliniennes, ou hitlériennes. La promotion de la Méthode Naturelle pourrait aller de pair avec la promotion des idées fascistes. Cela ne serait pas surprenant dans la mesure où elle s'inscrit originellement dans la perspective eugéniste, de Galton, Carrel et Richet, positions revendiquées généralement par les fascismes.

On ne peut nier toutefois que cet encrage profond des conceptions est probablement lié à l'industrialisation rapide et à l'urbanisation rapide des états européens particulièrement mais aussi peut-être des villes brésiliennes, à ce moment précis.

\section{Conclusion}

Les villes, au Brésil, se reconstruisent une pseudo nature très culturelle et civilisée, rationalisée, au travers de parcs, jardins, parfois à la française, lacs, etc. La vision de la nature y reste ambiguë, paradoxale. S'il s'agit de la nature champêtre, paysanne, rurale, sylvestre, elle peut être associée à l'image, par exemple, de Jeca Tatu, et dans 
ce cas être très dévalorisée. Tempérée de science, de technologie, artificielle elle devient positive pour les élites urbaines. La nature sauvage, peuplée d'animaux dangereux et d'indiens parfois hostiles, à l'inverse, ne peut qu'effrayer ou repousser. La réception pour le moins mitigée de la méthode Hébert pourrait s'expliquer par cette ambivalence et ambiguité.

Un mouvement similaire existe aussi en Europe et en France, plus précisément, mais, dans ce dernier pays, la nature ne fait plus peur depuis des lustres. Elle n'inquiète plus. Elle est presque trop civilisée, trop cultivée et de ce fait familière. Elle est d'autant plus familière que beaucoup de familles dans la dynamique du développement industriel et du travail des mines ou de l'industrie viennent de la quitter récemment, souvent une génération avant. Il y a donc une profonde nostalgie de celle-ci. C'est cette nostalgie qui pourrait expliquer sans doute, tout autant que l'eugénisme présent dans le champ intellectuel à l'époque, l'intérêt pour la Méthode Naturelle et plus généralement pour la nature. La nature dans ce cas n'est pas une nature artificielle et urbaine, même si celle-ci existe aussi, mais une nature «naturelle » présente non loin des villes et que la méthode Hébert propose d'investir.

Au Brésil, bien au contraire, la Méthode Naturelle ne saurait trouver une fécondité importante. En effet, elle est considérée, généralement, selon les sources consultées, comme insuffisamment scientifique, rationnelle et idéologique, ses bases étant trop précaires. La fécondité de la Méthode Naturelle en France est liée à un point de vue assez opposé. C'est l'excès de culture et de rationalité qui dérange, dans le domaine des pratiques corporelles (les gymnastiques trop mécaniques).

$\mathrm{Au}$ Brésil, ce qui conduit à une résistance réside dans le fait que la nature rurale ne correspond pas assez à l'idéal scientifique voire scientiste qui baigne les cercles intellectuels et les élites urbaines. Une nature artificielle et urbanisée (parcs, lacs artificiels, etc...) est davantage valorisée. «L'ordre et le progrès », emblèmes de ce pays, figurant sur son drapeau (ordem e progresso), ne peuvent se satisfaire d'une Méthode Naturelle considérée comme ne reposant pas sur des bases scientifiques solides, sur une très forte rationalité. C'est pour cela que les méthodes plus construites, pensées comme plus scientifiques (suédoise, néo-suédoise, Demenij, etc.) y trouveront une plus grande fécondité et un plus grand intérêt. A cela, bien sûr, il faut ajouter le fait que la méthode Hébert est française et en tant que telle ne peut être que redéfinie et réorganisée pour constituer une méthode nationale brésilienne.

\section{Références brésiliennes}

Andrade, Mario de. Paulicéia Desvairada. São Paulo, SP: Círculo do Livro, 1986.

Almeida, Conceição M. R. As águas e a cidade de Belém do Pará: história, natureza e cultura material no século XIX. São Paulo, SP: Pontifícia Universidade Católica de São Paulo, 2010.

Berto, Rosianny C., e Omar Scheiner. Educação em Revista: Circulação de Saberes na Imprensa Periódica da Educação Física, 1932-1945. In: Proceedings of the 16th Brazilian Sports Science Congress and 3th International Sport Science Congress. Salvador, BH: Brazilian Sports Sciences College, 2009.

Castro, Fabio F. A cidade Sebastiana: Era da borracha, memória e melancolia numa capital da periferia da modernidade. Belém, PA: Lavor, 2010. 
Cunha, Luciana B. "As roupas Esportivas em Revista na Cidade de Belo Horizonte, 1929-1950 : Moldes, Recortes e Costuras”, Campinas, SP: Universidade Estadual de Campinas (UNICAMP), 2011.

Dalben, André. "Inezil Penna Marinho: Formação de um Intelectual da Educação Física Brasileira.” Movimento 17, no.1 (2011): 59-76.

Dalben, André. Educação do Corpo e vida ao Ar Livre: Natureza e Educação Física em São Paulo (1930-1945). Campinas, SP: Universidade Estadual de Campinas (UNICAMP), 2009.

Dalben, André e Kátia Dalailof. "Natureza Urbana: Parques Infantis e Escola ao Ar Livre em São Paulo (1930-1940)." Revista Brasileira de Ciências do Esporte 31, no.1 (2009): 163-177.

Danailof, Kátia. Crianças na Trama Urbana: as Práticas Corporais nos Parques Infantis da São Paulo dos Anos 1930, Campinas, SP: Universidade Estadual de Campinas (UNICAMP), 2006.

Fausto, Boris. “A Revolução de 1930.” In Brasil em Perspectiva, Editado por Carlos Guilherme Mota, 227-255. São Paulo, SP: Difel, 1984.

Goellner, Silvana V., e André Luiz S. Silva. Recônditos da Memória: o Acervo Pessoal de Inezil Penna Marinho. Porto Alegre, RS: Gênese, 2009.

Goellner, Silvana V. Bela, Maternal e Feminina. Imagens da Mulher na Revista Educação Physica. Campinas, SP: Unicamp, 1999.

Goellner, Silvana V. "O Método Francês e a Militarização da Educação Física na Escola Brasileira”. In Pesquisa Histórica na Educação Física Brasileira, Editado por Amarílio Ferreira Neto, 123-143. Vitória, ES: Centro de Educação Física e Desportos/UFES, 1996).

Góis Junior, Edivaldo. Os Higienistas e a Educação Física : a História dos seus Ideais. Rio de Janeiro, RJ: Universidade Gama Filho, 2000.

Ferreira Neto, Amarílio. A Pedagogia no Exército e na Escola: A Educação Física Brasileira (1880-1950). Aracruz, ES: FACHA, 1999.

Linhales, Meily A. A Escola e o Esporte : Uma História de Práticas Culturais. São Paulo, SP: Editora Cortez, 2009.

Lucena, Ricardo de F. O Esporte na Cidade. Campinas SP: Autores Associados, 2001.

Melo, Victor A. de. Cidade Sportiva: Primórdios do Esporte no Rio de Janeiro. Rio de Janeiro, RJ: Relume Dumará, 2001.

Melo, Victor A. de. História da Educação Física e do Esporte no Brasil : Panorama e Perspectivas. São Paulo, SP: Ibrasa, 1999.

Moreno, Andrea. Corpo e Ginástica num Rio de Janeiro : Mosaico de Imagens e Textos. Campinas, SP: Unicamp, 2001.

Payer, Maria O. "O Rural no Espaço Público Urbano." In: Cidades Atravessadas: o Sentido Público no Espaço Urbano. Editado por Eni Puccinelli Orland, 165-174. Campinas, SP: Pontes, 2001.

Penna, Belisário. Saneamento do Brasil. 2th ed., Rio de Janeiro: Jacinto Ribeiro dos Santos, 1923.

Quitzau, Evelise A. Educação do corpo e vida associativa: as sociedades ginásticas alemãs em São Paulo (fins do século XIX primeiras décadas do século XX). Campinas, SP: Universidade Estadual de Campinas (UNICAMP), 2011.

Quitzau, Evelise Amgarten e Carmen Lúcia Soares. “A Força da Juventude Garante o Futuro de um Povo : A Educação do Corpo no Sport Club Germania (18991938)". Movimento 16, (2010):87-106. 
Schneider, Omar e Amarilio Ferreira Neto. "Americanismo e a Fabricação do 'Homem Novo': circulação e apropriação de modelos culturais na Revista Educação Physica (1932-1945)." Movimento 14, no. 1 (2008): 135-159.

Schneider, Omar. "Estratégias Editoriais, Enciclopedismo, Produtos e Publicidade na Revista Educação Physica (1932-1945).” Movimento 10, no. 3 (2004): 23-52.

Schossler, Joana Carolina. História do Veraneio no Rio Grande do Sul. Porto Alegre, RS: 2013.

Moraes e Silva, Marcelo. Novos modos de olhar, outras maneiras de se comportar: a emergencia do dispositivo esportivo da cidade de Curitiba, 1899-1919. Campinas, SP: Universidade Estadual de Campinas (UNICAMP), 2011.

Silva, Ana Márcia, Célia Guimarães Perini e Márcia de Souza Pedroso Agostini. "História de Bugres e Tigres: Corpo e Natureza em Terras Catarinenses," Pro-Posições, 14, no.2 (2003): 121-146.

Soares, Carmen L. "Corps, Éducation, Savoirs et Pratiques." In: Sport and Nature in History: Proceedings of the 7th ISHPES Congress, Montpellier, France, 26-31 August, 2001, Edited by Jean-Michel Delaplace, Sylvain Villaret and William Chameyrat, 180-185. Sankt Augustin: Academia Verlag, 2004.

Soares, Carmen L. "Georges Hébert e o Método Natural: Nova Sensibilidade, Nova Educação do Corpo." Revista Brasileira de Ciências do Esporte, 25. no. 1 (2003): 21-40.

Soares, Carmen L. "Corps, Éducation, Savoirs et Pratiques." In: Résumés - Abstracts of the VII Congrès de la Société Internationale d'Histoire du Sport et de l'Education Physique (Sport et nature dans l'histoire). ISHPES - AFRAPS Université Montpellier 1, Montpellier, França, 26 a 31 de agosto, 2001.

Soares, Carmen L. Imagens da Educação no Corpo: Estudo a partir da Ginástica Francesa no Século XIX. Campinas, SP: Editora Autores Associados, 1998.

Soares, Carmen L. Educação Física : Raízes Européias e Brasil. Campinas, SP: Editora Autores Associados, 1994.

Sola, Lourdes. "O Golpe de 1937 e o Estado Novo." In Brasil em Perspectiva, editado por Carlos Guilherme Mota, 256-282. São Paulo, SP: Difel, 1984.

\section{Sources brésiliennes consultées}

\section{Livres}

Azevedo, Fernando. Da Educação Física: o que Ela É, o que Tem Sido e o que Deveria Ser. 3th ed., São Paulo, SP: Melhoramentos, 1960.

Bonorino, Laurentino L. et al. Histórico da Educação Física. Vitória, ES: Imprensa Oficial, 1931.

Kretzschmar, Lotte. Cultura Physica Feminina. Rio de Janeiro, RJ: A Noite, 1932.

Miranda, Nicanor. A Harmonia entre o Corpo e o Espírito. Rio de Janeiro, RJ: Imprensa Nacional, 1945.

Marinho, Inezil P. Sistemas e Métodos de Educação Física. 5th ed., Rio de Janeiro, RJ: DEF-MES, 1952.

Pauchet, Victor. Conservai a Mocidade. São Paulo, SP: Civilização Brasileira, 1952.

Ramos, Jair J. Os Exercícios Físicos na História e na Arte. São Paulo, SP: Ibrasa, 1982. 
Annales de congrès

Congresso Nacional de Educação, 7th, 1935, Rio de Janeiro, Anais do Congresso Nacional de Educação, Rio de Janeiro, RJ: ABE, 1935.

Congresso Paulista de Educação Fisíca 1th, 1940, São Paulo. Anais do I Congresso Paulista de Educação Física, São Paulo, SP: APEF-SP, 1942.

Congresso Pan-Americano de Educação Física, 1th, 1943, Rio de Janeiro. Anais do I Congresso Pan-Americano de Educação Física, Rio de Janeiro, RJ: DEF, 1943.

\section{Revues}

Educaçao Physica (1932-1945)

Desbonnet, Edmundo. "Crítica ao Método Natural de Georges Hébert." Educação Physica 38, (1940): 33-36.

Hébert, Georges. “Os perigos Morais do Esporte.” Educação Physica 56, (1941): 30$32 ; 57-59$.

Hébert, Georges. "Os perigos Sociais do Esporte.” Educação Physica 57, (1941): 10$11 ; 66-67$.

Hébert, Georges. "Os Perigos Físicos do Esporte: Erros e Preconceitos Concernentes à Especialização.” Educação Physica 58, (1941): 10-11; 61-63.

Hébert, Georges. "Expressões Técnicas de Educação Física - Distinções entre 'Esporte', 'Educação Física', e 'Ginástica'.” Educação Physica 61, (1941): 10$11 ; 61-62$.

Hébert, Georges. "O Esporte dentro da Educação Física." Educação Physica 62, (1942): 12-13; 48.

Hébert, Georges. "Deveres Físicos Gerais do Homem.” Educação Physica 76, (1943): 27-28.

Revista de Educaçao Fisica - Exército - (1932-1949)

Vida e Saúde (1938-1949)

Viver ! (1938-1942)

Ouvrages de Georges Hébert dans les archives de la

Bibliothèque de l'Ecole d'Education Physique et de sport de l'Université de Sao Paolo. (Biblioteca da Escola de Educação Física e Esportes da USP) :

Hébert, Georges. Education Physique ou l'Entrainement Complet par la Méthode Naturelle: Historique Documentaire. Paris: Vuibert, 1947.

Hébert, Georges. Code de la Force. 7th ed., Paris: Vuibert, 1947.

Hébert, Georges. Sport contre l'Education physique. 4th ed., Paris: Vuibert, 1946.

Hébert, Georges. Guide Abrégé du Moniteur et de la Monitrice. 8th ed., Paris: Vuibert, 1946.

Hébert, Georges. Les Champs d'Ébats: Centre de Régénération, Virile et Morale: une Euvre Féconde et de Haute Portée Sociale. Paris: Vuibert, 1944.

Hébert, Georges. Guide Pratique d'Education physique. 4th ed., Paris: Vuibert, 1922.

Hébert, Georges. Lecon-Type d'Entrainement, Complet et Utilitaire. 4th ed., Paris: Vuibert, 1913. 


\section{Références et sources françaises}

Andrieu, Gilbert. "A la Recherche d'un Modèle ou Deux de Surhomme: le Gladiateur Combattant et l'Hercule Farnèse." In L'Education Physique au XXe Siècle Edité par Bernard-Xavier René, 227-239. Paris: EPS, 1992.

Andrieu, Gilbert. “Georges Hébert (1875-1957)." In Le Corps en Mouvement, Edité par Pierre Arnaud, 183-192. Toulouse: Privat, 1985.

Arnaud, Pierre. Le Corps en Mouvement, Toulouse: Privat, 1985.

Bui-Xuân, Gilles. "Les Écoles Michelin Optent pour la Méthode Naturelle." In Education Physique et Sport en France, Edité par Pierre Arnaud et Jean-Paul Clément et Michel Herr, 49-61. Clermont: AFRAPS, 1989.

Bui-Xuân, Gilles et Jacques Gleyse. L'Emergence de l'Education Physique. Georges Demenij, 1850-1917, et Georges Hébert, 1875-1957. Paris: Hatier, 2001.

Coll. Encyclopédie des sports. Paris: Larousse, 1924.

David, Pierre-Roger. "Défense de la Méthode Naturelle." Education Physique 29, (1962): 17

Delaplace, Jean-Michel. Georges Hébert. Sculpteur de Corps. Paris: Vuibert, 2005.

During, Bertrand. La Crise des Pédagogies Corporelles. Paris: Scarabée, CEMEA, 1981.

Gleyse, Jacques. Archéologie de l'Education Physique au XXe Siècle en France, Paris: PUF et l'Harmattan, 1995, 2th ed. 2006.

Gleyse, Jacques. L'Instrumentalisation $d u$ Corps. Une Archéologie de la Rationalisation Instrumentale $d u$ Corps de l'Age Classique à l'Époque Hypermoderne. Paris: L'Harmattan, 1997.

Hébert, Georges. Education Physique ou l'Entrainement Complet par la Méthode Naturelle. Historique Documentaire. Paris: Vuibert, 1942.

Labbé, Marcel. Traité d'Education Physique. Paris : Doin, 1930.

Loisel, Ernest. Les Bases Psychologiques de l'Education Physique. Paris: Nathan, 1935.

Michelin et Cie. Euvres Sociales de Michelin et Cie. Clermont-Ferrand: Michelin, 1927.

Rauch, André, Le Corps en Education physique. Paris : PUF, 1982.

Rauch, André. Le Souci du Corps. Paris: PUF, 1983.

Rauch, André, Vacances et pratiques corporelles. Paris : PUF, 1989.

Rousseau, Jean-Jacques. Émile ou de l'Education. Paris: Garnier, 1953 ( $1^{\text {re }}$ édition 1762).

Scharagrodski, Pablo. La Invenciòn del 'Homo Gymnasticus'. Buenos Aires: Prometeo, 2011.

Terret, Thierry. Les Jeux Interalliés de 1919: Sport, Guerre et Relations Internationales. Paris: L'Harmattan, 2002.

Vigarello, Georges et Michèle Métoudi. "La Nature et l'Air du Temps.” In : Travaux et Recherches en E.P.S., no. 6 (1980): 20-28.

Villaret, Sylvain et Jean-Michel Delaplace. « La Méthode Naturelle de Georges Hébert ou 'l'École Naturiste' en Education physique, 1900-1939. » STAPS 63, no. 1 (2004): 29-44.

Vuibert, Paul. La Méthode Naturelle du Lieutenant de Vaisseau Georges Hébert. Paris: Vuibert, 1921.

Vuillemin, Roger. La Méthode Naturelle d'Hébert. Paris: Grandes Editions Françaises, 1948. 


\section{Notes}

1. Meily Assbú Linhales. A Escola e o Esporte : Uma História de Práticas Culturais. (São Paulo SP: Editora Cortez, 2009).

2. José Murilo de Carvalho. Forças Armadas e Política no Brasil. (Rio de Janeiro RJ: Zahar, 2005).

3. Meily Assbú Linhales. A Escola e o Esporte : Uma História de Práticas Culturais. (São Paulo SP: Editora Cortez, 2009).

4. Meily Assbú Linhales. A Escola e o Esporte : Uma História de Práticas Culturais. (São Paulo SP: Editora Cortez, 2009); Carmen Lúcia Soares. Educação Física : Raizes Européias e Brasil. (Campinas SP: Editora Autores Associados, 1994); Carmen Lúcia Soares. Imagens da Educação no Corpo: Estudo a partir da Ginástica Francesa no Século XIX. (Campinas SP: Editora Autores Associados, 1998); Edivaldo Góis Junior. Os Higienistas e a Educação Física : a História dos seus Ideais, (Rio de Janeiro RJ: Universidade Gama Filho, 2000).

5. Carmen Lúcia Soares. Educação Física : Raizes Européias e Brasil. (Campinas SP: Editora Autores Associados, 1994).

6. Victor Andrade de Melo. História da Educação Física e do Esporte no Brasil : Panorama e Perspectivas. (São Paulo SP: Ibrasa, 1999).

7. Silvana Vilodre Goellner. O Método Francês e a Militarização da Educação Física na Escola Brasileira. in Pesquisa Histórica na Educação Física Brasileira, ed. Amarílio Ferreira Neto (Vitória ES: Centro de Educação Física e Desportos/UFES, 1996), 123-143.

8. Amarílio Ferreira Neto. A Pedagogia no Exército e na Escola: A Educação Física Brasileira (1880-1950). (Aracruz ES: FACHA, 1999).

9. Andrea Moreno. Corpo e Ginástica num Rio de Janeiro : Mosaico de Imagens e Textos, (Campinas SP: Unicamp, 2001).

10. Ana Márcia Silva, Célia Guimarães Perini and Márcia de Souza Pedroso Agostini. História de Bugres e Tigres: Corpo e Natureza em Terras Catarinenses," Pro-Posições, 14, 2 (2003): 121-146.

11. Meily Assbú Linhales. A Escola e o Esporte : Uma História de Práticas Culturais. (São Paulo SP: Editora Cortez, 2009).

12. Evelise Amgarten Quitzau. Educação do corpo e vida associativa: as sociedades ginásticas alemãs em São Paulo (fins do século XIX primeiras décadas do século XX), Ano de Obtenção: 2011. Universidade Estadual de Campinas, UNICAMP, Brasil; Evelise Amgarten Quitzau and Carmen Lúcia Soares. A Força da Juventude Garante o Futuro de um Povo : A Educação do Corpo no Sport Club Germania (1899-1938), Movimento 16, (2010):87-106.

13. André Rauch, Le Souci du Corps (Paris: PUF, 1983).

14. Voir à ce sujet Bancel Nicolas \& alii Zoos humains. Au temps des exhibitions humaines. Paris, La Découverte, 2004.

15. Jean-Michel Delaplace, Georges Hébert: Sculpteur de Corps (Paris : Vuibert, 2005), 100.

16. Caïmans, panthère tâchetée, anacondas...

17. André Dalben, Educação do Corpo e Vida ao Ar Livre: Natureza e Educação Física em São Paulo, 1930-1945, (Campinas SP: Unicamp, 2009), 33-47.

18. Carmen L. Soares, Georges, 21-40.

19. Pablo Scharagrodski, La Invenciòn del 'Homo Gymnasticus' (Buenos-Aires: Prometeo, 2011). 
20. Sur ce thème voir : André Dalben, Educação do Corpo e Vida ao Ar Livre: Natureza e Educação Física em São Paulo, 1930-1945, (Campinas SP: Unicamp, 2009), 33-47. Maria Onice Payer, "O Rural no Espaço Público Urbano," in Cidades Atravessadas: o Sentido Público no Espaço Urbano, ed. Eni Puccinelli Orland (Campinas: Pontes, 2001), 165-174.

21. Jeca Tatu est présenté au cours du XXe siècle comme l'image archétypale du paysan brésilien asthénique, apathique, plus ou moins touché par des maladies endémiques (fièvres, malaria) et surtout, paresseux, improductif. On le représente un chapeau sur les yeux, faisant plus moins la sieste sur le pas de porte de sa cabane insalubre. Mais, il finira sa vie, bien nourri et propriétaire d'une maison bourgeoise salubre et propre.

22. Mario de Andrade, Paulicéia Desvairada, (São Paulo, SP: Círculo do Livro, 1986 ).

23. André Dalben e Kátia Danailof, "Natureza Urbana: Parques Infantis e Escola ao Ar Livre em São Paulo, 1930-1940," Revista Brasileira de Ciências do Esporte 31, no.1 (2009): 163-177; André Dalben, Educação do Corpo e Vida ao Ar Livre: Natureza e Educação Física em São Paulo, 1930-1945, (Campinas SP: Unicamp, 2009); Kátia Danailof. Crianças na Trama Urbana: as Práticas Corporais nos Parques Infantis da São Paulo dos Anos 1930, (Campinas SP: Unicamp, 2006).

24. Ricardo de Figueiredo Lucena. O Esporte na Cidade, (Campinas SP: Aurores Associados, 2001); Victor Andrade de Melo. Cidade Sportiva: Primórdios do Esporte no Rio de Janeiro, (Rio de Janeiro RJ: Relume Dumará, 2001).

25. Luciana Bicalho da Cunha. As roupas Esportivas em Revista na Cidade de Belo Horizonte, 1929-1950 : Moldes, Recortes e Costuras, (Belo Horizonte MG: UFMG, 2011).

26. Marcelo Moraes e Silva. Novos modos de olhar, outras maneiras de se comportar: a emergencia do dispositivo esportivo da cidade de Curitiba, 1899-1919, (Campinas SP: Unicamp, 2011).

27. Joana Carolina Schossler. História do Veraneio no Rio Grande do Sul, (Porto Alegre RS: 2013).

28. Fabio Fonseca de Castro. A cidade Sebastiana: Era da borracha, memória e melancolia numa capital da periferia da modernidade. (Belém: Lavor, 2010); Conceição Maria Rocha de Almeida. As águas e a cidade de Belém do Pará: história, natureza e cultura material no século XIX. (São Paulo SP: Pontifícia Universidade Católica de São Paulo, 2010).

29. Régis Hébert, Témoignage de Georges Hébert, Officier du Suchet, communiqué par son fils Régis Hébert, Généalogie et Histoire de la Caraïbe, 148, (2002): 3513.

30. Georges Hébert, Education Physique ou l'Entrainement Complet par la Méthode Naturelle. Historique Documentaire (Paris: Vuibert, 1942).

31. Laurentino Lopes Bonorino e outros, Histórico da Educação Física (Vitória, ES: Imprensa Oficial, 1931).

32. Bonorino, 165-166.

33. Fernando de Azevedo. Da Educação Física: o que Ela É, o que Tem Sido e o que Deveria Ser. (São Paulo SP: Melhoramentos, 1960).

34. Inezil Penna Marinho, Sistemas e Métodos de Educação Física (Rio de Janeiro RJ: DefMes, 1952).

35. La dictature de "l'Etat Nouveau" a duré de 1937 à 1945. Sur ce thème voir: Boris Fausto, "A Revolução de 1930" in Brasil em Perspectiva, ed. Carlos Guilherme Mota (São Paulo, SP: Difel, 1984), 227-255. Lourdes Sola, "O Golpe de 1937 e o Estado Novo" in Brasil em Perspectiva, ed. Carlos Guilherme Mota (São Paulo, SP: Difel, 1984); entre autres. Sur Inezil Penna Marinho et ses rapports avec l'Etat Nouveau, voir : André Dalben, "Inezil Penna Marinho: Formação de um Intelectual da Educação Física Brasileira." Movimento 17, no.1 (2011): 59-76. Silvana V. Goellner and André Luiz S. Silva, Recônditos da Memória: o Acervo Pessoal de Inezil Penna Marinho (Porto Alegre: Gênese, 2009). 
36. Nicanor Miranda, A Harmonia entre o Corpo e o Espírito (Rio de Janeiro RJ: Imprensa Nacional, 1945).

37. Miranda, 55.

38. Miranda, 52.

39. Miranda, 55.

40. Lotte Kretzschmar, Cultura Physica Feminina (Rio de Janeiro RJ: A Noite, 1932).

41. Georges Hébert, L'Éducation Physique Féminine: Muscle et Beauté Plastique (Paris: Vuibert, 1919).

42. Jean-Michel Delaplace, Georges Hébert. Sculpteur de corps (Paris: Vuibert, 2005).

43. Gilles Bui-Xuân et Jacques Gleyse, L'Emergence de l'Education physique. Georges Demenij, 1850-1917, et Georges Hébert, 1875-1957, (Paris: Hatier, 2001).

44. Sylvain Villaret et Jean-Michel Delaplace, « La Méthode Naturelle de Georges Hébert ou 'l'école naturiste' en Education Physique, 1900-1939, » STAPS 63, no. 1 (2004): 29-44.

45. Pierre Arnaud, Le Corps en Mouvement, (Toulouse : Privat, 1985).

46. Gilbert Andrieu, "A la Recherche d'un Modèle ou Deux de Surhomme : Le Gladiateur Combattant et l'Hercule Farnèse," in L'Education Physique au XXe Siècle, ed. Bernard-Xavier René (Paris: EPS, 1992), 227-239.

47. Congrès International de l'Education Physique, 17-20 Mars 1913, (v.3 Paris, Baillères, 1913) : 9; Congrès International de l'Education Physique, 17-20 Mars 1913, (v.1 Paris, Baillères, 1913) : 20.

48. Omar Schneider e Amarílio Ferreira Neto, « Americanismo e a Fabricação do 'Homem Novo': Circulação e Apropriação de Modelos Culturais na Revista Educação Physica, 19321945, » Movimento 14, no.1 (2008): 135-159

49. Sur l'Etat Nouveau au Brésil voir note numéro 33 de cet article.

50. Voir à ce sujet les analyses de Silvana V. Goellner, Bela, Maternal e Feminina: Imagens da Mulher na Revista Educação Physica (Campinas, SP: Unicamp, 1999), 6-7.

51. Voir, par exemple, la Revista Educação Physica 17, (1938): 24-26.

52. Schneider e Ferreira Neto.

53. Thierry Terret, Les Jeux Interalliés de 1919: Sport, Guerre et Relations Internationales (Paris: L'Harmattan, 2002).

54. Par ordre chronologique les articles sont :

Georges Hébert, “Os Perigos Morais do Esporte.” Educação Physica 56, (1941): 3032, 57-59. (Les dangers moraux du sport); Georges Hébert, "Os Perigos Sociais do Esporte." Educação Physica 57, (1941): 10-11, 66-67. (Les dangers sociaux du sport) ; Georges Hébert, "Os Perigos Físicos do Esporte: Erros e Preconceitos Concernentes à Especialização." Educação Physica 58, (1941): 10-11, 61-63. (Les dangers physiques du sport: erreurs et préjugés concernant la spécialisation); Georges Hébert, "Expressões Técnicas de Educação Física - Distinções entre 'Esporte', 'Educação Física', e 'Ginástica'.” Educação Physica 61, (1941): 10-11, 61-62. (Expressions et techniques d'Education Physique - distinction entre «sport» et «Education Physique » et "gymnastique »); Georges Hébert, "O Esporte dentro da Educação Física." Educação Physica 62, (1942): 12-13, 48. (Le sport dans l'Education physique) ; Georges Hébert, "Deveres Físicos Gerais do Homem." Educação Physica 76, (1943): 27-28. (Les devoirs physiques généraux de l'homme).

55. Georges Hébert, Le Sport Contre l'Education physique. (Paris: Vuibert, 1946; 1th. ed.1925) 
56. Georges Hébert, La Culture Virile et les Devoirs Physiques de l'Officier Combattant (Paris: G. Oudin, 1913).

57. Carmen L. Soares, "Corps, éducation, savoirs et pratiques," in Résumés - Abstracts of the VII Congrès de la Société Internationale d'Histoire du Sport et de l'Education Physique (Sport et nature dans l'histoire), ed. ISHPES - AFRAPS (Montpellier: ISHPES, 2001).

58. Carmen L. Soares, "Georges Hébert e o Método Natural: Nova Sensibilidade, Nova Educação do Corpo," Revista Brasileira de Ciências do Esporte, 25. no.1 (2003): 21-40.

59. Carmen L. Soares, "Corps, Éducation, Savoirs et Pratiques," in Proceedings of the 7th Congrès de la Société Internationale d'Histoire du Sport et de l'Education Physique (Sport et Nature dans l'Histoire), ed. Jean-Michel Delaplace and Sylvain Villaret and William Chameyrat (Sankt Augustin: Academia Verlag, 2004), 180-185.

60. Rosianny C. Berto e Omar Schneider, "Educação em Revista: Circulação de Saberes na Imprensa Periódica da Educação Física, 1932-1945," in Proceedings of the 16th Brazilian Sports Science Congress and 3th International Sport Science Congress, ed. Brazilian Sports Sciences College (Salvador BH: Brazilian Sports Sciences College, 2009).

61. Sur ce thème et ce médecin voir: Dalben, Educação, 33-47.

62. Sur ce thème voir: André Dalben e Kátia Dalailof, "Natureza Urbana: Parques Infantis e Escola ao Ar Livre em São Paulo, 1930-1940," Revista Brasileira de Ciências do Esporte 31, no.1 (2009): 163-177.

63. L'Humanité, 28 avril 1913, 2

64. Voir à ce sujet André Rauch Les Savoirs du corps, Paris, PUF, 1983, Le Corps en Education Physique, Paris, PUF, 1982 et Vacances et pratiques corporelles, Paris, PUF, 1989.

65. Voir Gilles Bui-Xuân, "Les Écoles Michelin Optent pour la Méthode Naturelle," in Education Physique et Sport en France, 1920-1980, ed. Pierre Arnaud and Jean-Paul Clément and Michel Herr (Clermont: AFRAPS, 1989): 49-61.

66. Pierre Arnaud \& alii Le Sport et les français pendant l'occupation. Tome 1, (Paris: L'harmattan, 2002) : 54.

67. Jacques Gleyse, "Les méthodes actives ("l'Education Nouvelle") en Education physique et sportive, STADION, International Journal of the History of Sport, 37, no.1, (2011): 15-31. 


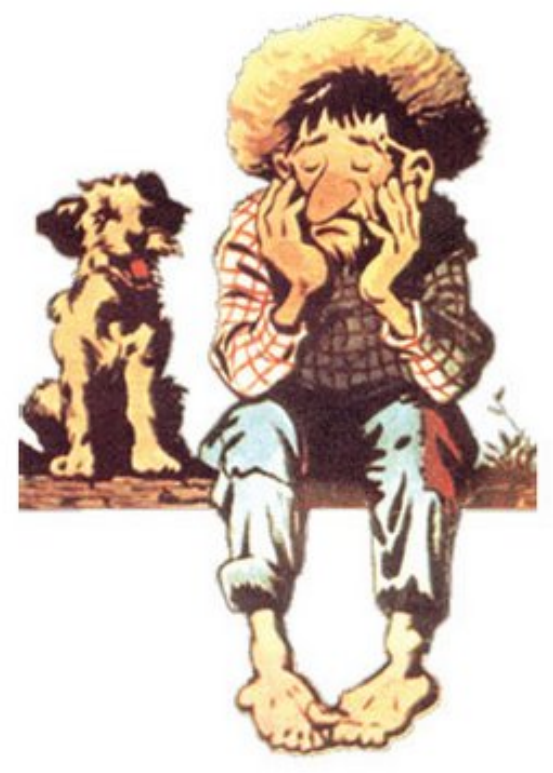

Figure 1 - Libre de droits, Jeca Tatu. 


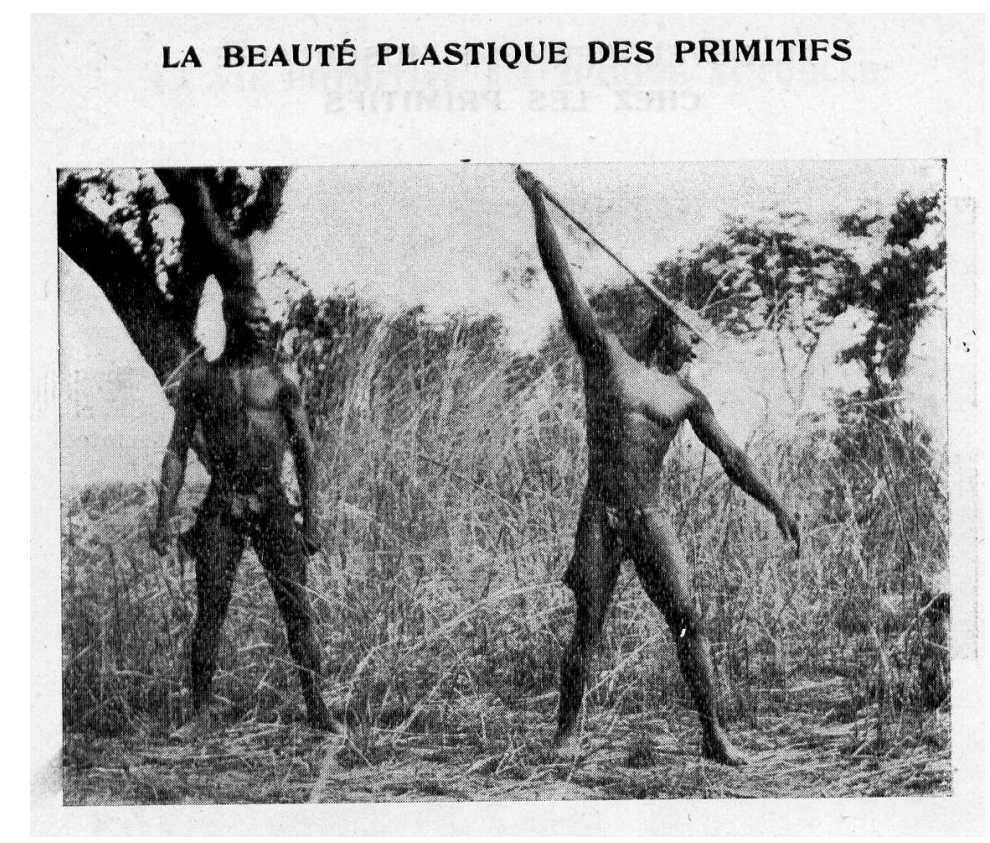

Figure 4 
UN Geste de PORTAGe RENOUVELÉ dE L'ANTIQUe

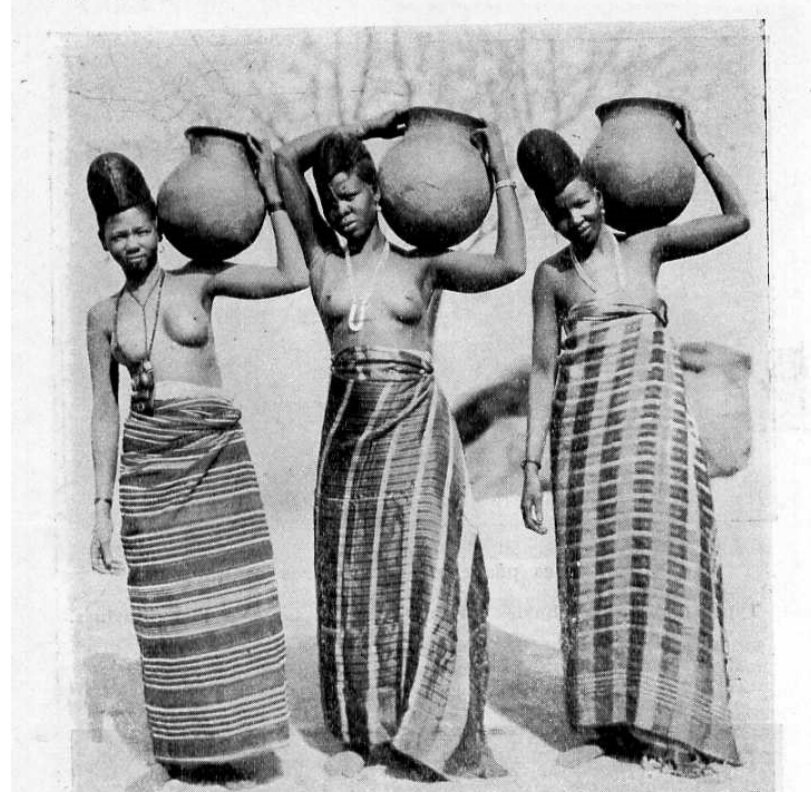

Figure 5 - Issue de L'Education Physique virile et morale par la Méthode Naturelle, Tome 1, 1936. 